\title{
Probabilistische Modellierung und Vorhersage der Standzeit und Zuverlässigkeit eines Fräswerkzeugs mittels der Bayesianischen Statistik
}

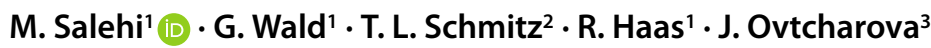 \\ Eingegangen: 27. März 2019 / Angenommen: 4. Dezember 2019 / Online publiziert: 27. Januar 2020 \\ (c) Der/die Autor(en) 2019
}

\section{Zusammenfassung}

In diesem Artikel wird die probabilistische Modellierung und Vorhersage für die Standzeit und Zuverlässigkeit eines Fräswerkzeugs mittels der Bayesianischen Statistik beschrieben. Zur Entwicklung des probabilistischen Modells wird eine Markov-Chain-Monte-Carlo-Simulation (MCMC) auf das Taylor-Standzeit-Modell angewendet. Die A-priori-Wahrscheinlichkeitsverteilungen werden aus der Literaturrecherche ermittelt. Aus anschließenden Fräsversuchen werden ein Trainings- und ein Testdatensatz generiert. Die Versuche werden in einem Bereich der Schnittgeschwindigkeiten von 300 bis $400 \mathrm{~m} / \mathrm{min}$ durchgeführt. Die A-posteriori-Wahrscheinlichkeitsverteilungen werden unter Verwendung des Trainingssatzes berechnet. Mittels der trainierten A-posteriori-Wahrscheinlichkeitsverteilungen werden anschließend die Testdatensätze bei den Schnittgeschwindigkeiten von 300 bis $400 \mathrm{~m} / \mathrm{min}$ vorhergesagt. Es folgt ein Vergleich des probabilistischen Standzeitmodells mit den Testdatensätzen. Anschließend werden die A-posteriori-Verteilungen der Werkzeugstandzeit verwendet, um eine Zuverlässigkeitsanalyse unter Verwendung der Zuverlässigkeitsfunktion in Abhängigkeit der Schnittgeschwindigkeit durchzuführen. Es wird gezeigt, dass die Werkzeugstandzeit- und Zuverlässigkeitsfunktion die gemessenen Werkzeugstandzeiten innerhalb der Unsicherheitsintervalle mit einem maximalen Vorhersagefehler von $18 \%$ mit lediglich zwei Trainingsdatenpunkten vorhersagen können.

Schlüsselwörter Bayesianische Statistik · MCMC · Werkzeugstandzeit · Werkzeugzuverlässigkeit · Taylor-Modell

\section{Probabilistic modeling and prediction of a milling tool life and reliability using bayesian statistics}

\begin{abstract}
This paper represents probabilistic modeling and prediction for lifetime and reliability of a milling tool using Bayesian statistics. Markov Chain Monte Carlo (MCMC) simulation is applied to Taylor tool life model to develop probabilistic models. Prior probabilities are established from literature review and posterior probabilities of the Taylor model parameters are obtained using milling experiments. The experiments were conducted to train and test the probabilistic models under a range of cutting speeds, $300-400 \mathrm{~m} / \mathrm{min}$. In this regard, the training datasets are used to update the tool life model and the test datasets are used to validate the probabilistic tool life model. Posterior distributions of the tool life are used to perform reliability analysis using reliability functions. The probabilistic models investigate the effect of cutting speeds on tool life probability distributions and the reliability functions. It is shown that the tool life posterior and reliability functions predict the measured tool life values within the uncertainty intervals and maximum prediction error of $18 \%$, using only two training data points.
\end{abstract}

Keywords Bayesian inference $\cdot$ MCMC $\cdot$ Tool life $\cdot$ Tool reliability $\cdot$ Taylor model

M. Salehi

mehdi.salehi@hs-karlsruhe.de

1 Institute of Materials and Processes, Hochschule Karlsruhe - Technik und Wirtschaft, Moltkestr. 30, 76133 Karlsruhe, Deutschland
2 Mechanical, Aerospace, and Biomedical Engineering, University of Tennessee, Knoxville, Knoxville, TN 37996, USA

3 Institut für Informationsmanagement im Ingenieurwesen, Karlsruher Institut für Technologie, Kriegsstr. 77, 76133 Karlsruhe, Deutschland 


\section{Einleitung}

Die Werkzeugstandzeit ist ein wichtiges Fertigungsmerkmal für die Bearbeitungsleistung und beeinflusst erheblich die Werkstückqualität und Fertigungsproduktivität. In diesem Zusammenhang wurden mehrere Leistungsmodelle für die Vorhersage der Werkzeugstandzeit und dessen Verschleiß entwickelt. Diese Modelle können in analytische, numerische, empirische, auf künstlicher Intelligenz (KI) basierende und hybride Modelle klassifiziert werden [1-4]. Unter den empirischen Modellen wurde das TaylorModell definiert, um die Werkzeugstandzeit vorherzusagen. Es beschreibt einen empirischen Zusammenhang zwischen der Werkzeugstandzeit und der Schnittgeschwindigkeit anhand des Potenzgesetzes

$\mathrm{T}=\left(\frac{\mathrm{C}}{V_{c}}\right)^{\frac{1}{n}}$.

Dabei ist $T$ die Werkzeugstandzeit in Minuten, um eine Verschleißmarkenbreite $V_{B}$ von beispielsweise $V_{B}=0,3 \mathrm{~mm}$ zu erreichen. $V_{c}$ ist die Schnittgeschwindigkeit in $\mathrm{m} / \mathrm{min}$ und $C$ ist eine empirisch ermittelte Konstante, auch Minutengeschwindigkeit genannt. Dazu werden die Standzeiten $T$ bei unterschiedlichen Schnittgeschwindigkeiten $V_{c}$ empirisch ermittelt und daraus der Taylorexponent $n$ bestimmt. Die Konstante $C$ beschreibt theoretisch die Schnittgeschwindigkeit bei einer Standzeit $T=1 \mathrm{~min}$. Die Vorgehensweise wird in $[5,6]$ näher beschrieben. Die Werkzeugstandzeit, die zum Erreichen einer vorbestimmten Freiflächen- oder Kolkverschleißgrenze erforderlich ist, wird als Schneidzeit definiert. Der Freiflächenverschleiß ist aufgrund seines vorhersagbaren und regelmäßigen Verschleißmusters das häufigste und bevorzugte Verschleißkriterium.

Der Werkzeugverschleiß variiert in realen Anwendungen, selbst wenn das Schneidwerkzeug, die Maschine und die Schneidbedingungen identisch sind. Dies kann auf die Variation der chemischen und physikalischen Eigenschaften des kommerziell identischen Werkstücks und auf die Werkzeug-zu-Werkzeug-Leistung zurückzuführen sein. Im Allgemeinen ist die Standzeit des Werkzeugs durch $\mathrm{Zu}-$ fälligkeit gekennzeichnet. Eine exakte Vorhersage ist daher schwierig. Deterministische Modelle sind demnach in der Anwendung begrenzt, da die Zufälligkeit der Ergebnisse der Werkzeugstandzeit nicht bewertet wird [7, 8]. Dies führt dazu, dass stochastische Größen genutzt werden können, um diese Streuung für identische Standzeitversuche zu berücksichtigen. Basierend auf diesem Hintergrund wurden die Unsicherheitsquantifizierung und die probabilistische Vorhersage der Werkzeugstandzeit mit Bayesianischen Statistiken durchgeführt. Niaki et al. [9, 10] entwickelte probabilistische Modelle unter Verwendung der Bayesianischen Methode, um den Werkzeugverschleiß beim Fräsen von Nickelbasiswerkstoffen vorherzusagen. Dabei wurde ein kombinierter Gibbs-Metropolis-Algorithmus angewendet, um die unbekannten Parameter eines nichtlinearen mechanistischen Schnittleistungsmodells abzuschätzen. Unter Verwendung des Metropolis-Algorithmus zur Vorhersage der Modellparameter und der Gibbs-Sampler zur Aktualisierung der Messfehlerabweichung wurden die Modellparameter mit einem maximalen Fehler von $18 \%$ vorhergesagt.

Unter der Zuverlässigkeit eines Systems versteht man im Allgemeinen die Zeitdauer in der das System seine Funktion erfüllt und nicht ausfällt [11]. Die Bestimmung der Werkzeugstandzeit unter Prozessunsicherheiten kann durch die Anwendung von Zuverlässigkeitstechniken berechnet werden. Somit können auch Ausfallwahrscheinlichkeiten von Schneidwerkzeugen mit den Zuverlässigkeitsfunktionen vorhergesagt werden. Die Zuverlässigkeitsanalyse der Standzeit und des Verschleißes von Schneidwerkzeugen wurde bereits mit verschiedenen Wahrscheinlichkeitsverteilungen und statistischen Methoden untersucht $[12,13]$. Lin [14] hat die grundlegende Zuverlässigkeitsanalyse von Schneidwerkzeugen beim Hochgeschwindigkeitsfräsen anhand von experimentellen Tests untersucht. Er verglich die Ergebnisse der Zuverlässigkeitsfunktionen unter Berücksichtigung unterschiedlicher Schnittgeschwindigkeiten und Vorschübe. Elbestawi et al. [15] hat stochastische Modelle zur Vorhersage der Ausfallrate beim Drehen von gehärtetem Stahl mit keramischen Werkzeugen vorgestellt. Verschiedene Arten des Werkzeugverschleißes, einschließlich des gleichmäßigen und chemischen Verschleißes und des vorzeitigen Ausfalls, wurden unter Verwendung von Lognormal- und Weibull-Verteilungen modelliert. Diese quantifizieren das Ausfallverhalten des Werkzeugs bei unterschiedlichen Schnittgeschwindigkeiten anhand der Zuverlässigkeits- und Wahrscheinlichkeitsdichtefunktionen (WDF), siehe Abschn. 3.2. Es wurde gezeigt, dass die logarithmische Normalverteilung als Werkzeugverschleißverteilung verwendet werden kann, um die Werkzeugausfallrate unter verschiedenen Werten der Schnittbedingungen und der Oberflächengüte des Werkstücks vorherzusagen. Dabei wurde die Weibull-Verteilung als die geeignetste Verteilung bewertet, um den Microbruch des Werkzeugs im Speziellen zu modellieren.

Die in den oben genannten Forschungen durchgeführte Zuverlässigkeitsanalyse beruht auf datengesteuerten Analysen. Diese wurden mit verschiedenen Schneidbedingungen und Werkzeuggeometrien anhand umfangreicher Experimente ermittelt. Eine alternative Methode zur Bestimmung der Wahrscheinlichkeitsverteilung und der Zuverlässigkeitsfunktion der Werkzeugstandzeit ist die Bayesianische Statistik, bei der eine anfängliche „Meinung“(Vorwissen) über Modellparameter der Werkzeugstandzeitmodelle in die aktuelle und zukünftige Analyse integriert werden kann. Das Vorwissen (A-priori-Wahrscheinlichkeit) über die Mo- 
Abb. 1 Verschleißmessungsvorgang: Fräswerkzeug und Mikroskop (a) und Bearbeitungssetup (b)
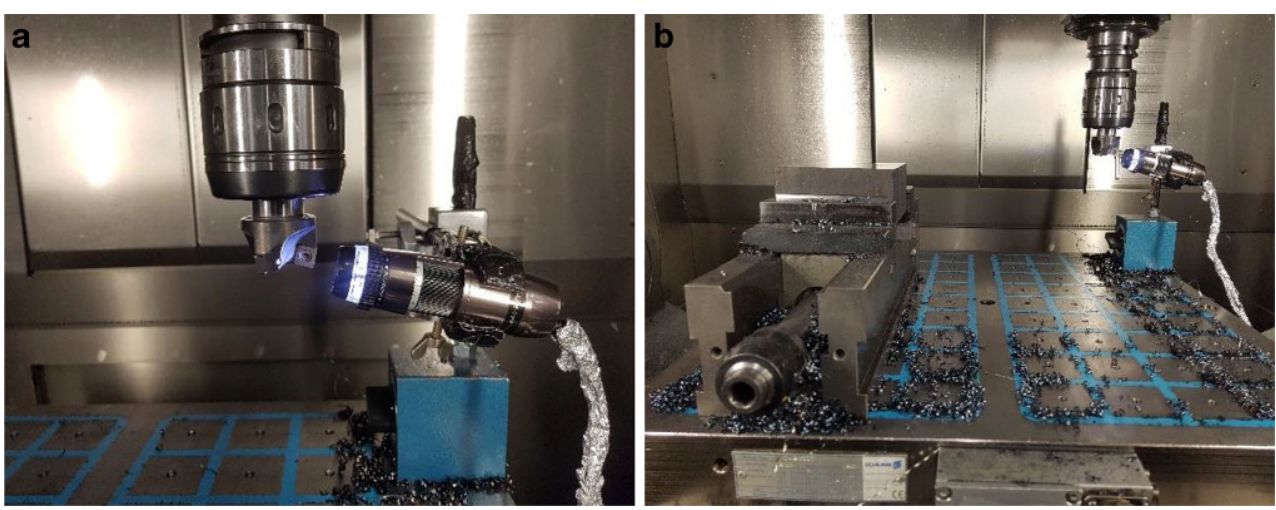

dellparameter kann von ehemaligen Experimenten oder einer Literaturrecherche stammen. Dadurch können die Modellparameter mit deutlich geringerem experimentellen Aufwand bestimmt werden.

Im Folgenden wird die Bayesianische MCMC-Methode für die probabilistische Vorhersage der Werkzeugstandzeit und der Zuverlässigkeit beim Fräsen modelliert und angewendet. Die Normalverteilung wird als Wahrscheinlichkeitsverteilungsfunktion und als Zuverlässigkeitsfunktion verwendet. Darüber hinaus wird der Einfluss der Schnittgeschwindigkeit auf die Standzeit und Zuverlässigkeit des Fräswerkzeugs anhand der probabilistischen Modelle untersucht.

\section{Versuchsaufbau und empirische Datengewinnung}

Zur Aktualisierung und abschließenden Validierung der probabilistischen Modellierung und Vorhersage der Standzeit und Zuverlässigkeit eines Fräswerkzeugs mittels der Bayesianischen Statistik müssen zunächst anwendungsspezifische Versuchsdaten ermittelt werden. Dazu wurden Fräsversuche auf einer 5-Achs-Fräsmaschine Hermle C40 durchgeführt; siehe Abb. 1. Für die 2,5 achsigen Versuche wurde als Fräswerkzeug ein Werkzeughalter mit drei

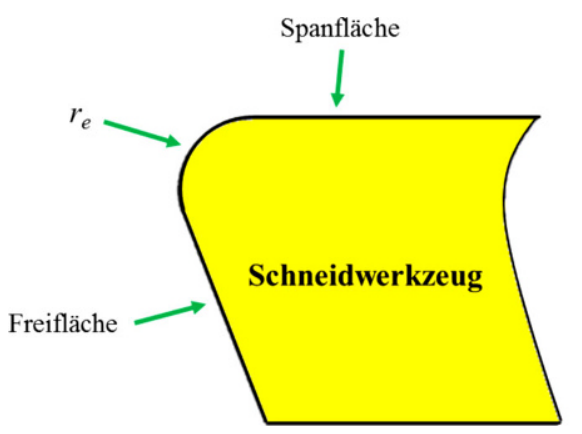

Abb. 2 Skizze für den Schneideinsatz SPGW09T308 unbeschichteten Wendeschneidplatten mit der kommerziellen Bezeichnung SPGW09T308 und Kantenradien von $r_{e}=20 \mu \mathrm{m}$ bei einem Werkzeugdurchmesser von $32 \mathrm{~mm}$ verwendet, siehe Abb. 2. Die Wendeschneidplatten wurden von der Zermet Zerspanung GmbH mit dem ISO-Schneidstoff P25 konstruiert und hergestellt. Als Versuchswerkstoff diente der kubische Werkzeugstahl AISI 1045 mit den Abmessungen $100 \times 100 \times 100 \mathrm{~mm}$. Die Schnitttiefe betrug $b=1,5 \mathrm{~mm}$ bei einem Vorschubwert von $f_{z}=0,05 \mathrm{~mm} / \mathrm{Zahn}$ und vier Schnittgeschwindigkeiten von $V_{c}=\{300 ; 325 ; 350$; $400\} \mathrm{m} / \mathrm{min}$.

Um ein Entfernen des Werkzeugs zu Messzwecken von der Frässpindel zu vermeiden, wurde ein tragbares Mikroskop 5 Mpx Dino-Lite (AM7915MZT) mit der maximalen Vergrößerung von 220 verwendet. In regelmäßigen Abständen wurden damit Aufnahmen der Werkzeugfreifläche durchgeführt. Die Werkzeugfreiflächenverschleißmarkenbreite $V_{B}$ als Werkzeugverschleißkriterium wurde für drei Wendeplatten nach jedem Intervall von $200 \mathrm{~mm}$ Schnittlänge gemessen und der jeweilige Mittelwert und die Standardabweichung der abgenutzten Wendeplatten bis zum Erreichen des Freiflächenverschleißkriteriums von $V_{B}=0,3 \mathrm{~mm}$ ermittelt.

Abb. 3 zeigt exemplarisch die Aufnahmen des Werkzeugverschleißwachstums bei einer Schnittgeschwindigkeit von $V_{c}=350 \mathrm{~m} / \mathrm{min}$, welche in 5 Schritten aufgenommen

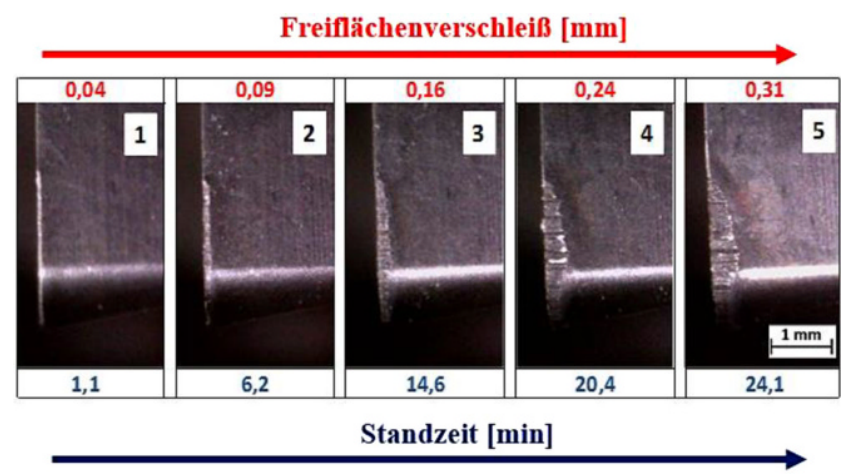

Abb. 3 Messung des durchschnittlichen Freiflächenverschleißes des Fräswerkzeugs mit dem Mikroskop 


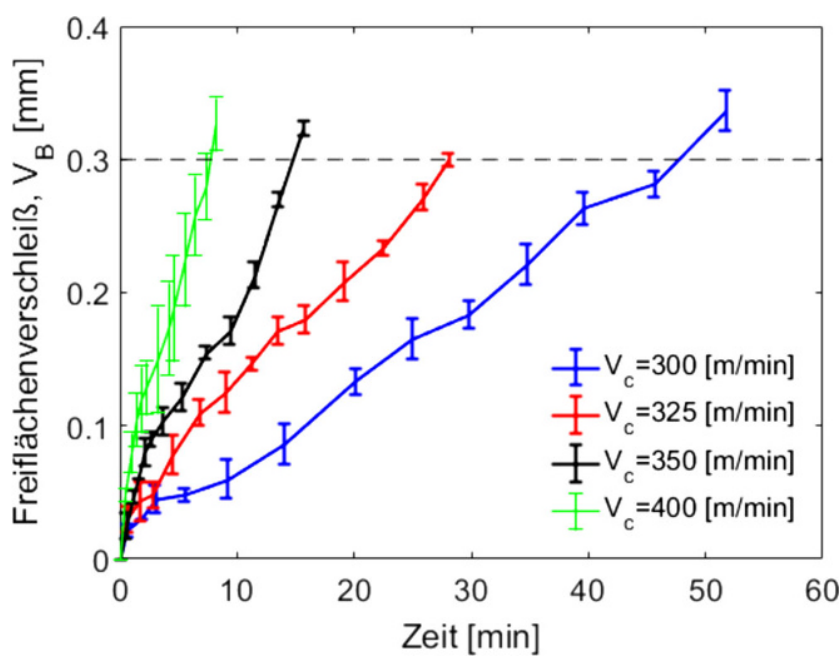

Abb. 4 Werkzeugfreiflächenverschleiß bei vier gewählten Schnittgeschwindigkeiten

wurden. Zur Bestimmung der Werkzeugstandzeit wurde eine lineare Interpolation zwischen benachbarten Datenpunkten durchgeführt, bis die Verschleißgrenze von $0,3 \mathrm{~mm}$ erreicht wurde. In Abb. 4 sind die Ergebnisse der Werkzeugverschleißversuche dargestellt, wobei jeder Datenpunkt den Mittelwert der drei Versuche zusammen mit einem Standardabweichungsfehlern von $\pm 1 \sigma$ angegeben wird. Der Freiflächenverschleiß steigt mit der Zerspanungszeit. Die Standzeit verringert sich mit einer Erhöhung der Schnittgeschwindigkeit von 300 auf $400 \mathrm{~m} / \mathrm{min}$. Die gestrichene Linie stellt das Werkzeugverschleißkriterium (Freiflächenverschleiß) $V_{B}=0,3 \mathrm{~mm}$ dar. Der gemessene Mittelwert und die Standardabweichung von $1 \sigma$ der Werkzeugstandzeit für die jeweiligen Schnittgeschwindigkeiten beim Erreichen des Verschleißkriteriums wird in Tab. 1 gezeigt.

\section{Methodik}

Die probabilistische Modellierung der Werkzeugstandzeit wird unter Verwendung der Bayesianischen Inferenz auf das Taylor-Modell durchgeführt. Zunächst werden die A-priori-Wahrscheinlichkeiten der Modellparameter festgelegt. Folgend werden die Modellparameter unter Verwen-

Tab. 1 Mittelwerte und $1 \sigma$ Standardabweichungen der Werkzeugstandzeit bei einem durchschnittlichen Freiflächenverschleiß von $0,3 \mathrm{~mm}$

\begin{tabular}{lll}
\hline No & $V_{c}(\mathrm{~m} / \mathrm{min})$ & $T_{- \text {gemessene }}(\min )$ \\
\hline 1 & 300 & $(48 ; 0,9)$ \\
2 & 325 & $(28 ; 0,4)$ \\
3 & 350 & $(14,8 ; 0,55)$ \\
4 & 400 & $(7,6 ; 0,35)$ \\
\hline
\end{tabular}

dung der Ergebnisse der durchgeführten Versuche mittels der Likelihood-Funktion aktualisiert, um die A-posterioriWahrscheinlichkeiten der Parameter des Taylor-Modells zu berechnen. Anschließend wird der Markov-Chain-MonteCarlo-Ansatz (MCMC-Ansatz) zur Simulation angewendet und die Parameter der A-posteriori-Wahrscheinlichkeitsverteilungen approximiert. Die A-posteriori-Wahrscheinlichkeitsverteilungen dienen schließlich als Startwerte der Berechnung der Zuverlässigkeitsfunktionen.

\subsection{Bayesianische Inferenz des Taylor- Standzeitmodells}

Die Bayesianische Inferenz ermöglicht die Anpassung eines A-priori-Wertes (eine anfängliche Annahme eines Parameters) durch das Einbeziehen neuer experimenteller Ergebnisse. Nach Gl. (2) ist die A-posteriori-Wahrscheinlichkeit $p(x \mid y)$ das Produkt der A-priori-Verteilung $p(x)$ mit der Likelihood-Funktion $p(y \mid x)$, dividiert durch die Normalisierungsfunktion. Unter Verwendung des Bayesianischen Ansatzes kann die A-posteriori-Verteilung einer Untersuchung als die A-priori-Verteilung einer zweiten Untersuchung verwendet werden $[16,17]$.

$p(x \mid y)=\frac{p(x) p(y \mid x)}{\int p(x) p(y \mid x) d x}$

Das Integral in Gl. (2) wird oft als marginale Wahrscheinlichkeit bezeichnet und hat im Allgemeinen keine geschlossene Lösung. Daher wurden Berechnungsansätze, u. a. die Markov-Chain-Monte-Carlo(MCMC)-Simulation, entwickelt, um die analytische Integration zu ergänzen oder $\mathrm{zu}$ ersetzen und die A-posteriori-Verteilung zu approximieren [18].

\subsubsection{Identifikation der Taylor-Modellparameter}

Die probabilistische Modellierung der Werkzeugstandzeit unter Verwendung der Normalverteilung beginnt mit der Festlegung der Taylor-Modellparameter $C$ und $n$. Die A-priori-Wahrscheinlichkeit der Modellparameter $C$ und $n$ wird als gemeinsame Gaußsche Verteilung $p(C, n)$ dargestellt. Der Mittelwert und die Standardabweichung der Parameter wurden aus [5, 19] für einen kohlenstoffarmen Stahl entnommen:

- $C=340 \pm 60 \mathrm{~m} / \mathrm{min}( \pm 1 \sigma)$

- $n=0,26 \pm 0,05( \pm 1 \sigma)$

Die A-priori-Verteilungen werden unter Verwendung der Ergebnisse der Bearbeitungsversuche mit der LikelihoodFunktion aktualisiert. Die bivariate Likelihood-Funktion der 
gemessenen Werkzeugstandzeit unter Berücksichtigung der Parameter des Taylor-Modells ergibt sich dann wie folgt:

$p\left(T_{m} \mid C, n\right)=e^{-\frac{\left(\left(\frac{C}{V_{c}}\right)^{\frac{1}{n}}-T_{m}\right.}{2 \sigma_{T m}{ }^{2}}}$

$p\left(T_{m} \mid C, n\right)$ ist hier die Likelihood-Funktion der gemessenen Werkzeugstandzeit $T_{m}$ bei gegebenen A-priori-Werten der Taylor-Modellparameter $(C, n) . \sigma_{T m}$ ist die Standardabweichung der gemessenen Standzeit. Eine Normalisierung der Wahrscheinlichkeit ist hier nicht sinnvoll, da es sich nicht um eine Wahrscheinlichkeitsdichtefunktion der Modellparameter $(C, n)$ handelt, sondern um die Wahrscheinlichkeit über die gemessenen Standzeitdaten $T_{m}$. Diese Wahrscheinlichkeitsfunktion beschreibt wie wahrscheinlich das Messergebnis bei einer bestimmten Schnittgeschwindigkeit bei gegebenen Taylor-Modellparametern ist.

Die A-posteriori-Wahrscheinlichkeit wird nach der Aktualisierung der A-priori-Wahrscheinlichkeit unter Verwendung der Likelihood-Funktion berechnet. Die A-posterioriWahrscheinlichkeitsverteilung der Werkzeugstandzeit wird unter Verwendung der Normalverteilung berechnet und wie folgt beschrieben:

$p\left(C, n \mid T_{m}\right)=\frac{p(C, n) \cdot p\left(T_{m} \mid C, n\right)}{p\left(T_{m}\right)}$,

dabei ist $p\left(C, n \mid T_{m}\right)$ die gemeinsame A-posteriori-Verteilung der Parameter $(C, n)$, berechnet durch das Produkt der gemeinsamen A-priori-Verteilung $p(C, n)$ und der Likelihood-Funktion $p\left(T_{m} \mid C, n\right)$ dividiert durch den Normalisierungsfaktor $p\left(T_{m}\right)$. Die gemeinsame A-posteriori-Verteilung der Parameter ist die Zielverteilung und wird mittels MCMC-Simulation approximiert, um die Wahrscheinlichkeitsverteilung der Werkzeugstandzeit für die Gl. (1) zu berechnen.

\subsubsection{Anwendung von MCMC auf das Taylor-Modell}

Die MCMC-Methode ist i. A. eine probabilistische Methode zum Ziehen einer Stichprobe $x$ aus einer Wahrscheinlichkeitsverteilung durch einen Markov-Kettenmechanismus (Markov Chain). Die daraus entstandene Verteilung wird als Zielverteilung $p(x)$ approximiert [20]. Die MCMCSimulation wird hier verwendet, um die Parameter des Taylor-Modells $(C, n)$ vorherzusagen. Dabei wird der Metropolis-Algorithmus der MCMC-Methode verwendet, um eine Stichprobe aus der Vorschlagsverteilung $q(C, n) \mathrm{zu}$ ziehen und die A-posteriori-Zielverteilung $p(C, n)$ zu approximieren. Als Vorschlagsverteilung wird eine gemeinsame Normalverteilung der Taylor-Modellparameter $(C, n)$ festgelegt.
Die Zielverteilung $p(C, n)$ ist das Produkt der gemeinsamen A-priori-Verteilung und der Likelihood-Funktion. Gemäß dem Metropolis-Algorithmus wird der Nenner hier nicht berechnet. Es folgt eine aus der Vorschlagsverteilung entnommene Kandidatenstichprobe $(C, n)^{\text {neu }}$, welche entweder akzeptiert oder abgelehnt wird, abhängig von einem festzulegenden Akzeptanzverhältnis der Akzeptanzrate $r$. In jeder Iteration erfolgt eine Anpassung der Markov-Kette zu $(C, n)^{\text {neu }}$, wenn die Probe akzeptiert wird. Ansonsten bleibt die Kette auf dem aktuellen Wert von $(C, n)^{i}$. Der Algorithmus fährt mit $N$-1-Iterationen fort, um $N$ Stichproben aus der Zielverteilung unter Verwendung der folgenden Schritte zu erhalten:

1. Lege eine normale A-priori-Verteilung $p(C, n)$ fest;

2. Lege eine Vorschlagsverteilung $q(C, n)$ fest;

3. Initialisiere eine Stichprobe als Startwert $(C, n)^{0}$;

4. Beginne mit der Iteration von $i=0$ bis $i=N-1$ :

- Wähle eine Kandidatenstichprobe $(C, n)^{\text {neu }}$ aus der Vorschlagsverteilung $q\left((C, n)^{\text {neu }} \mid(C, n)^{i}\right)$;

- Berechne die A-posteriori-Verteilung $p\left((C, n)^{i} \mid T_{m}\right)=$ $p\left((C, n)^{i}\right) p\left(T_{m} \mid(C, n)^{i}\right)$;

- Berechne die Akzeptanzrate $r=\frac{p\left((C, n)^{\text {neu }}\right)}{P\left((C, n)^{i}\right)}$;

- Generiere eine Zufallszahl $u$ aus einer Gleichverteilung zwischen 0 und 1

Wenn $u \leq r$ :

Akzeptiere den Vorschlag: $(C, n)^{\mathrm{i}+1}=(C, n)^{\mathrm{neu}}$; andernfalls:

Lehne den Vorschlag ab: $(C, n)^{i}=(C, n)^{\text {neu}}$;

5. Ende der Iteration.

Um die übermäßige Autokorrelation aufgrund der $\mathrm{Ab}$ hängigkeit der mit dem Metropolis-Algorithmus gezogenen Proben zu reduzieren, wird die Ausdünnungstechnik verwendet [21]. Darüber hinaus wird die Vorschlagsverteilung der Proben innerhalb der festgelegten Akzeptanzrate von $15-50 \%$ begrenzt $[20,22,23]$. Die anfänglichen Iterationen zeigen verfahrensbedingt zunächst ein transientes Verhalten bis sie in einen stationären Bereich übergehen. Dies wird gewöhnlich als Einbrennperiode (Burn-in-Periode) bezeichnet. Um diesen Einfluss zu unterbinden, wird dieser Bereich der Iterationen verworfen, um die Auswirkungen der Anfangsfehler zu Beginn der Kette zu reduzieren [24].

Die Beobachtung der Histogramme der Parameter ist dabei eine Möglichkeit die Konvergenz zur stationären Verteilung der Kette zu bewerten [21]. Dazu wird die GewekeMethode verwendet. Dabei wird das letzte Intervall der Kette mit einem kleineren Intervall am Beginn der Kette, z. B. die ersten $10 \%$ und die letzten $50 \%$ nach dem Entfernen der Anfangsiterationen, verglichen. Sind die Mittelwerte von zwei Intervallen in einem definierten Toleranzbereich, befindet sich die Kette im stationären Zustand [25]. Die Er- 
gebnisse der Anwendung des beschriebenen Algorithmus werden in Kap. 4 dargestellt.

\subsection{Zuverlässigkeitsfunktion}

Die Zuverlässigkeitsfunktion $R(t)$ ist die häufigste verwendete Funktion bei der Analyse der Standzeitdaten im Ingenieurwesen. Diese Funktion gibt die Wahrscheinlichkeit an, dass ein Bauteil für eine bestimmte Zeit fehlerfrei funktioniert. Um die Zuverlässigkeitsfunktion für ein Schneidwerkzeug zu ermitteln, muss zunächst die kumulative Verteilungsfunktion $F(t)$ (Engl.: cumulative distribution function: CDF) des Werkzeugausfalls berechnet werden. Die CDF des Werkzeugausfalls bezieht sich auf die Wahrscheinlichkeit, dass ein Werkzeug zu dem Zeitpunkt $t$ verschlissen ist und berechnet sich wie folgt:

$F(t)=\int_{0}^{t} f(T) d T$

Hier ist $T$ die Zeit bei der ein Werkzeugausfall auftritt. Als Ausfallkriterium am Ende der Standzeit wird der Freiflächenverschleiß von $V_{B}=0,3 \mathrm{~mm}$ festgelegt. Die Lösung des Integrals ist nicht trivial und wird numerisch gelöst. Die Zuverlässigkeitsfunktion $R(t)$ ist schließlich das Komplement der CDF:

$R(t)=1-F(t)$

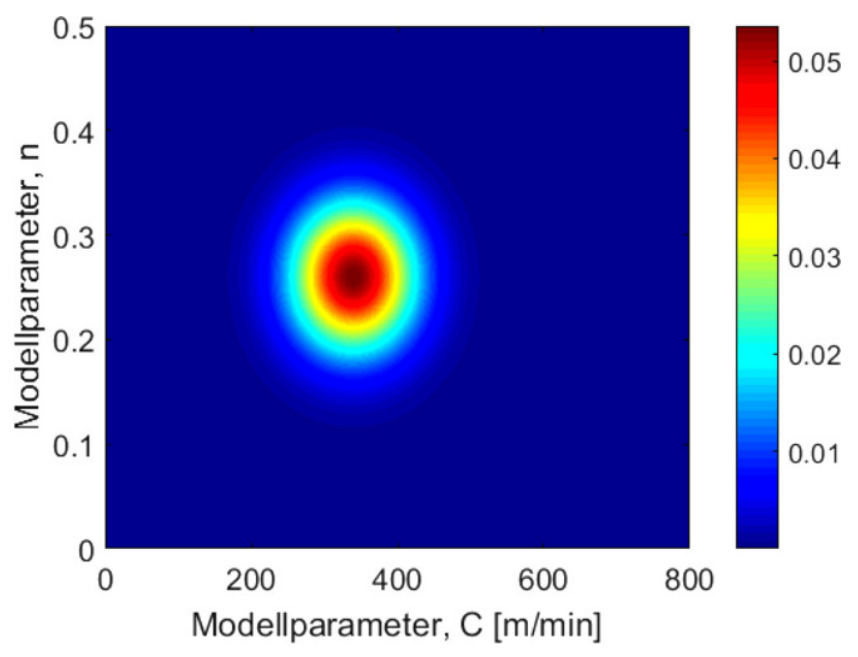

Abb. 5 Gemeinsame A-priori-Verteilungen der Modellparameter $C$ und $n$

\section{Ergebnisse der Werkzeugstandzeit- und Zuverlässigkeitsvorhersage}

In diesem Abschnitt werden die Ergebnisse der TaylorModellparameteridentifikation sowie die Vorhersage der Standzeit und Zuverlässigkeit der Werkzeuge unter Verwendung der Bayesianischen Statistik mittels MCMC-Simulation dargestellt. In diesem Zusammenhang werden die Parameter des Modells unter Verwendung von zwei Standzeitdatenpunkten $T_{m}=\{48 ; 7,6\}$ min bei den entsprechenden Schnittgeschwindigkeiten von $V_{c}=\{300 ; 400\} \mathrm{m} / \mathrm{min}$ aktualisiert. Das trainierte Modell wird dann verwendet, um die Testdatenpunkte bei den Schnittgeschwindigkeiten von $300 \mathrm{bis} 400 \mathrm{~m} / \mathrm{min}$ vorherzusagen.

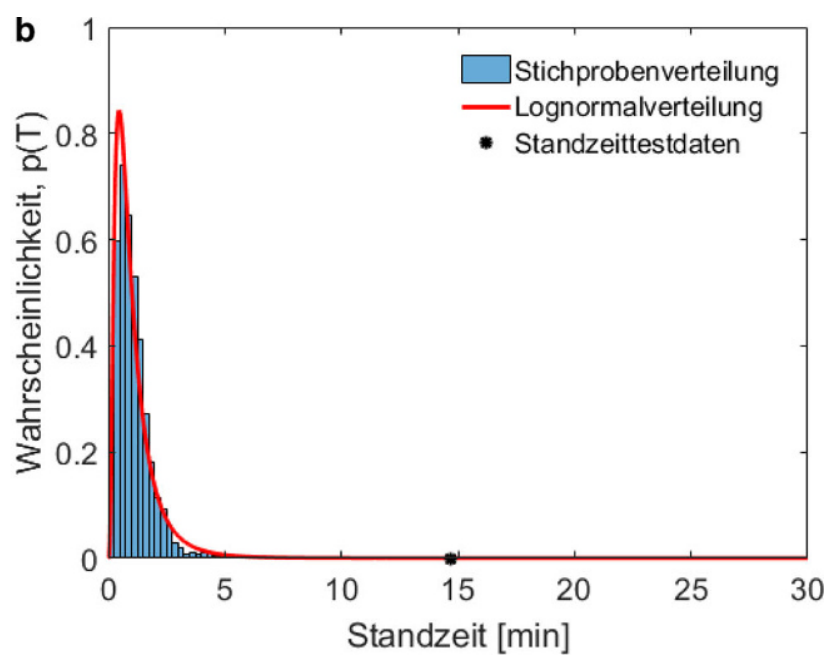

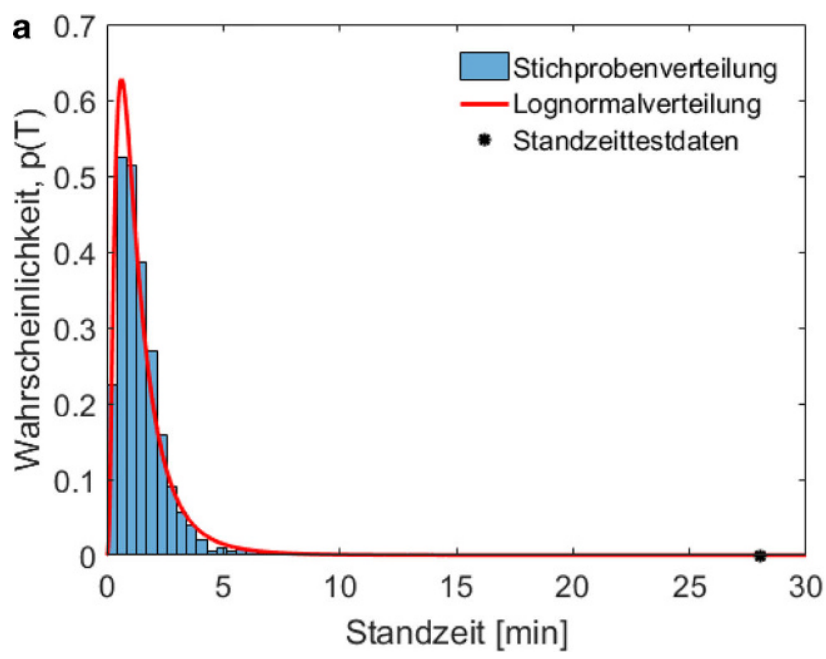

Abb. 6 Lognormal-A-priori-Funktionen mit den Schnittgeschwindigkeiten $325 \mathrm{~m} / \mathrm{min}$ (a) und 350 m/min (b) 


\subsection{Vorhersage der Werkzeugstandzeit}

Die modellierte gemeinsame A-priori-Verteilung der Modellparameter $p(C, n)$ beinhaltet zunächst eine Kovarianzmatrix, welche als unabhängig definiert wird, siehe Abb. 5 . Um die Korrelation der Parameter $C$ und $n$ zu ermitteln, wird der Korrelationskoeffizient bestimmt. Der Korrelationskoeffizient ist das Maß der linearen Beziehung zwischen zwei Parametern, die als Kovarianz der Parameter, dividiert durch das Produkt ihrer Standardabweichungen definiert sind und berechnet sich zu

$\rho(C, n)=\frac{\operatorname{cov}(C, n)}{\sigma_{C} \sigma_{n}}$.

Anschließend wird eine Monte-Carlo-Simulation durchgeführt, um die A-priori-Verteilung der Werkzeugstandzeit $T$ unter Verwendung der gemeinsamen A-priori-Verteilung von $C$ und $n$ zu bestimmen. Für die Simulation werden $N=3000$ zufällige $C$ - und $n$-Proben aus der gemeinsamen Vorverteilung für die Gl. (1) unter Verwendung der Schnittgeschwindigkeiten $V_{c}=\{325 ; 350\} \mathrm{m} / \mathrm{min}$ gezogen.

Abb. 6 zeigt das Histogramm der Werkzeugstandzeit (in Blau), die entsprechende logarithmische Normalverteilung (rote Linie) und die gemessene Werkzeugstandzeit (Standzeittestdaten) bei den genannten Schnittgeschwindigkeiten. Die Lognormalverteilung liefert dabei ein ausreichend gutes Approximieren an das Histogramm der Standzeit. Die dabei auftretende Rechtsschiefe-Verteilung (right skewed) basiert auf der Potenzgesetzform der Taylor-Gleichung. Die A-priori-Funktion kann zunächst die gemessene Werkzeugstandzeit aufgrund der wenig informativen A-priori-Werte für die Modellparameter $C$ und $n$, basierend auf der Literaturrecherche, nicht vorhersagen.

Es folgt die MCMC-Simulation zur Ermittlung der A-posteriori-Verteilung der Parameter $C$ und $n$. Für die Simulation werden 7500 Proben aus den Vorschlagsverteilungen $q(C, n)$ gezogen und die Wahrscheinlichkeitsfunktion aktualisiert. Abb. 7 zeigt das Kurvendiagramm mit dem ersichtlichen Übergang vom transienten in den stationären Bereich der Verteilungen für die ersten 1000 Stichproben des Parameters $n$. Diese werden als Burn-in-Periode aus der Simulation verworfen, um eine stationäre Verteilung der Stichproben zu gewährleisten.

Abb. 8 zeigt das Histogramm und die Wahrscheinlichkeitsverteilung der gezogenen Proben für den Parameter $n$. Die normale Wahrscheinlichkeitsverteilung (rote Farbe) wird an das Histogramm der gezogenen Proben angepasst. Die mit den MCMC-Algorithmen berechnete Akzeptanzrate der entnommenen Proben beträgt $r=0,34$. Abb. 8 zeigt das Trace-Diagramm der Kette, die sich nach dem Verwerfen der Burn-in-Periode im stationären Zustand befindet.

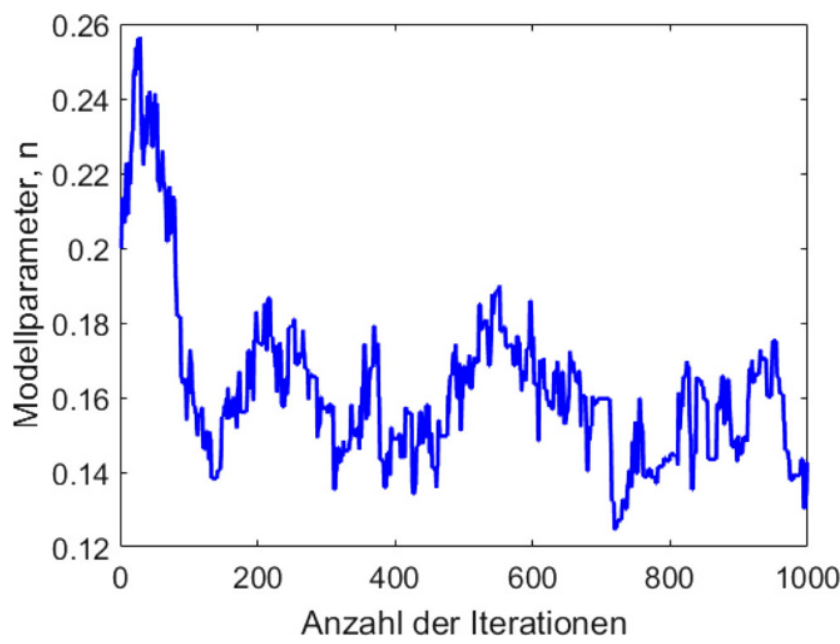

Abb. 7 Burn-in-Stichproben für den Parameter $n$
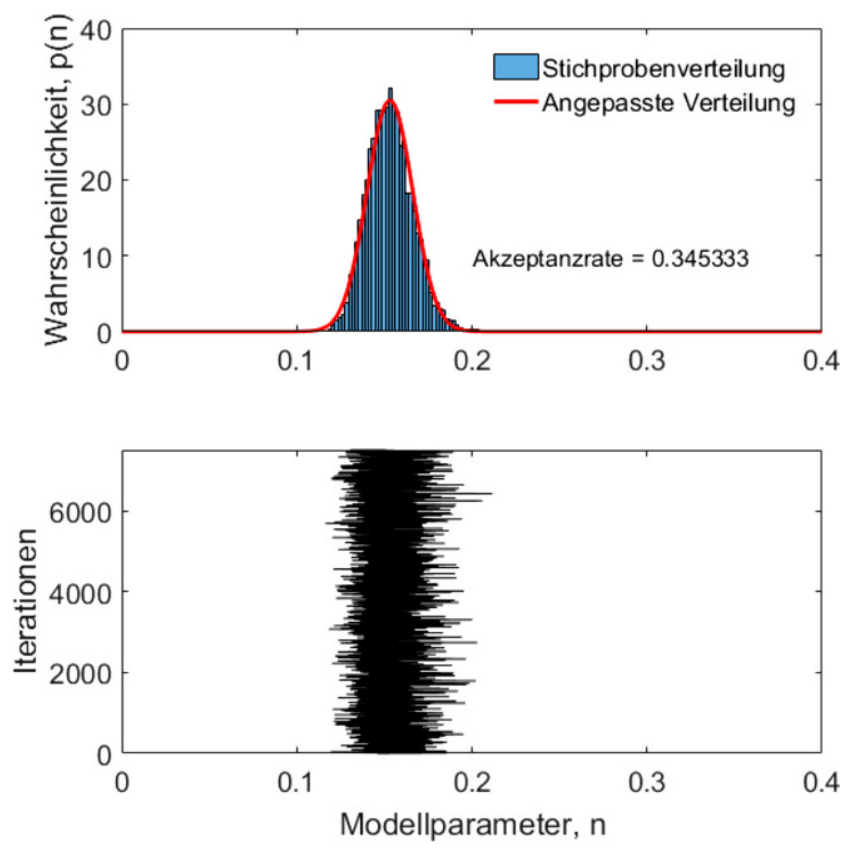

Abb. 8 Histogramm und Trace-Diagramm der A-posteriori-Stichprobe für den Parameter $n$

Eine gleichmäßige Verteilung der Stichproben ist damit sichergestellt.

Nach der Abstimmung der Kovarianzmatrix wird $K(C, n)$ für die Vorschlagsverteilung $q(C, n)$ als unabhängig festgelegt und wie folgt beschrieben:

$K_{(C, n)}=\left[\begin{array}{cc}300 & 0 \\ 0 & 7 e^{-5}\end{array}\right]$

Abb. 9 zeigt die gemeinsame A-posteriori-Verteilung der Parameter $C$ und $n$ unter Verwendung der zwei Aktualisierungsstandzeitdaten. Die Mittelwerte der Parameter $C$ und $n$ betragen $539 \mathrm{~m} / \mathrm{min}$ und 0,153 und die zugehöri- 


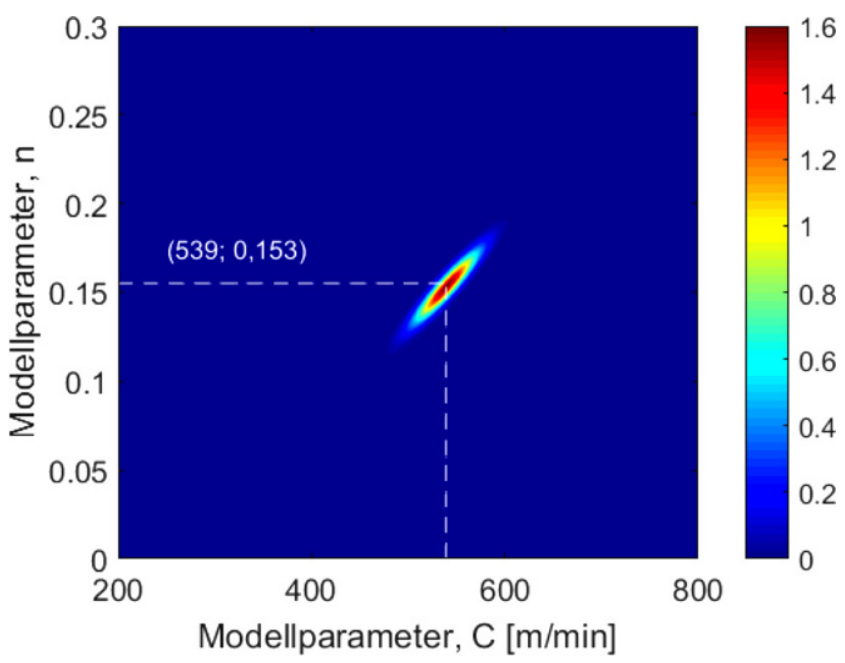

Abb.9 Gemeinsame A-posteriori-Verteilungen der Modellparameter $C$ und $n$

gen Standardabweichungswerte betragen $21,6 \mathrm{~m} / \mathrm{min}$ und 0,013. Vergleicht man die gemeinsame A-priori- (Abb. 5) und A-posteriori-Verteilungen (Abb. 9), zeigt sich eine Reduzierung der Unsicherheiten. Darüber hinaus korrelieren die Modellparameter $C$ und $n$ mit dem Korrelationskoeffizienten von 0,93. Der hohe Wert des Korrelationskoeffizienten gegen 1 beschreibt die starke Beziehung zwischen den beiden Parametern $C$ und $n$.

Die A-posteriori-Verteilungen der Standzeit werden unter Verwendung der Monte-Carlo-Simulation berechnet, indem die marginalen A-posteriori-Verteilungen von $C$ und $n$ in die Gl. (1) eingefügt werden. Abb. 10 zeigt die normalen A-posteriori-Verteilungen der Werkzeugstandzeit in roter Farbe mit dem Mittelwert und der Standardabweichung von $2 \sigma$ für die vier Schnittgeschwindigkeiten von $V_{c}=\{300 ; 325 ; 350 ; 400\} \mathrm{m} / \mathrm{min}$. Die A-priori-Verteilungen werden in den Abbildungen auch mit den Lognormalverteilungen in blauer Farbe dargestellt. Dabei zeigt sich, dass die gemessenen Werkzeugstandzeiten bei allen Schnittgeschwindigkeiten innerhalb des Unsicherheitsintervalls von $\pm 2 \sigma$ der A-posteriori-Funktionen liegen, die A-priori-Funk- tionen diese jedoch nicht vorhersagen können. Es ist wichtig zu beachten, dass die A-posteriori-Funktionen mit nur zwei Trainingsdatenpunkten aktualisiert wurden.

Die gemessenen und vorhergesagten Werkzeugstandzeiten werden zusätzlich zum Prozentsatz des Vorhersagefehlers in Tab. 2 angegeben. Die vorhergesagten Werte werden mit dem Mittelwert und einem Standardabweichungsintervall von $1 \sigma$ angegeben. Die prozentualen Fehlerwerte zwischen gemessener und vorhergesagter mittlerer Werkzeugstandzeit werden wie folgt berechnet:

$\%_{\text {error }}=\left|\frac{F_{\text {measured }}-F_{\text {predicted }}}{F_{\text {measured }}}\right| \cdot 100$.

Die ermittelten Fehler liegen dabei zwischen $0 \%$ bei einer Schnittgeschwindigkeit von $325 \mathrm{~m} / \mathrm{min}$ und $18 \%$ bei einer Schnittgeschwindigkeit von $350 \mathrm{~m} / \mathrm{min}$. Alle gemessenen Standzeitwerte liegen innerhalb der Unsicherheitsintervalle von $\pm 2 \sigma$ der vorhergesagten Verteilungen.

\subsection{Zuverlässigkeitsvorhersage}

Basierend auf den ermittelten Werkzeugstandzeiten wird gemäß Gln. (5) und (6) die Zuverlässigkeit vorhergesagt. Abb. 11 zeigt die Zuverlässigkeitsfunktionen $R(T)$ der Werkzeuge bei den Schnittgeschwindigkeiten von $V_{c}=\{300 ; 325 ; 350 ; 400\} \mathrm{m} / \mathrm{min}$ zusammen mit den gemessenen Werkzeugstandzeiten beim Erreichen der durchschnittlichen Freiflächenverschleißmarkenbreite von $V_{B}=0,3 \mathrm{~mm}$. Die berechneten Mittelwerte der Zuverlässigkeitsfunktionen bei dem Zuverlässigkeitswert von 0,5 entsprechen der vorhergesagten Standzeit bei jeder Schnittgeschwindigkeit.

Die Steigung der Zuverlässigkeitsfunktion wächst mit Zunahme der Schnittgeschwindigkeit von 300 bis $400 \mathrm{~m} / \mathrm{min}$. Je höher die Schnittgeschwindigkeit, desto höher ist die Abnahme der Zuverlässigkeit. Bei der Schnittgeschwindigkeit von $V_{c}=350 \mathrm{~mm} / \mathrm{min}$ kann die A-posterioriZuverlässigkeitsfunktion die gemessenen Datenpunkte im Vergleich zu den anderen A-posteriori-Funktionen nicht
Tab. 2 Schnittdaten, gemessene Standzeitdatenpunkte und Bayesianische Vorhersage

Tab. 3 Gemessene Standzeitdatenpunkte und die entsprechenden Zuverlässigkeitswerte

\begin{tabular}{lllll}
\hline No & $V_{c}(\mathrm{~m} / \mathrm{min})$ & $T_{\text {_gemessene }}(\min )$ & $T_{\text {_vorhergesagt }}(\mathrm{min})$ & $T_{\text {_Fehler }}(\%)$ \\
\hline 1 & 300 & 47,$1 ; 48 ; 48,9$ & $(46,7 ; 5,58)$ & 1,$2 ; 2,7 ; 4,5$ \\
2 & 325 & 27,$5 ; 28,2 ; 28,4$ & $(27,5 ; 2,67)$ & $0 ; 1,7 ; 3$ \\
3 & 350 & 14,$3 ; 14,8 ; 15,4$ & $(16,9 ; 1,53)$ & $18 ; 14,2 ; 9$ \\
4 & 400 & 7,$3 ; 7,7 ; 8$ & $(7,05 ; 0,84)$ & 3,$4 ; 7,2 ; 9$ \\
\hline
\end{tabular}

\begin{tabular}{llll}
\hline No & $V_{c}(\mathrm{~m} / \mathrm{min})$ & $T_{\text {gemessene }}(\min )$ & $R(t)$ \\
\hline 1 & 300 & 47,$1 ; 48 ; 48,9$ & 0,$47 ; 0,41 ; 0,35$ \\
2 & 325 & 27,$5 ; 28,2 ; 28,4$ & 0,$5 ; 0,4 ; 0,37$ \\
3 & 350 & 14,$3 ; 14,8 ; 15,4$ & 0,$95 ; 0,91 ; 0,84$ \\
4 & 400 & 7,$3 ; 7,7 ; 8$ & 0,$38 ; 0,22 ; 0,13$ \\
\hline
\end{tabular}



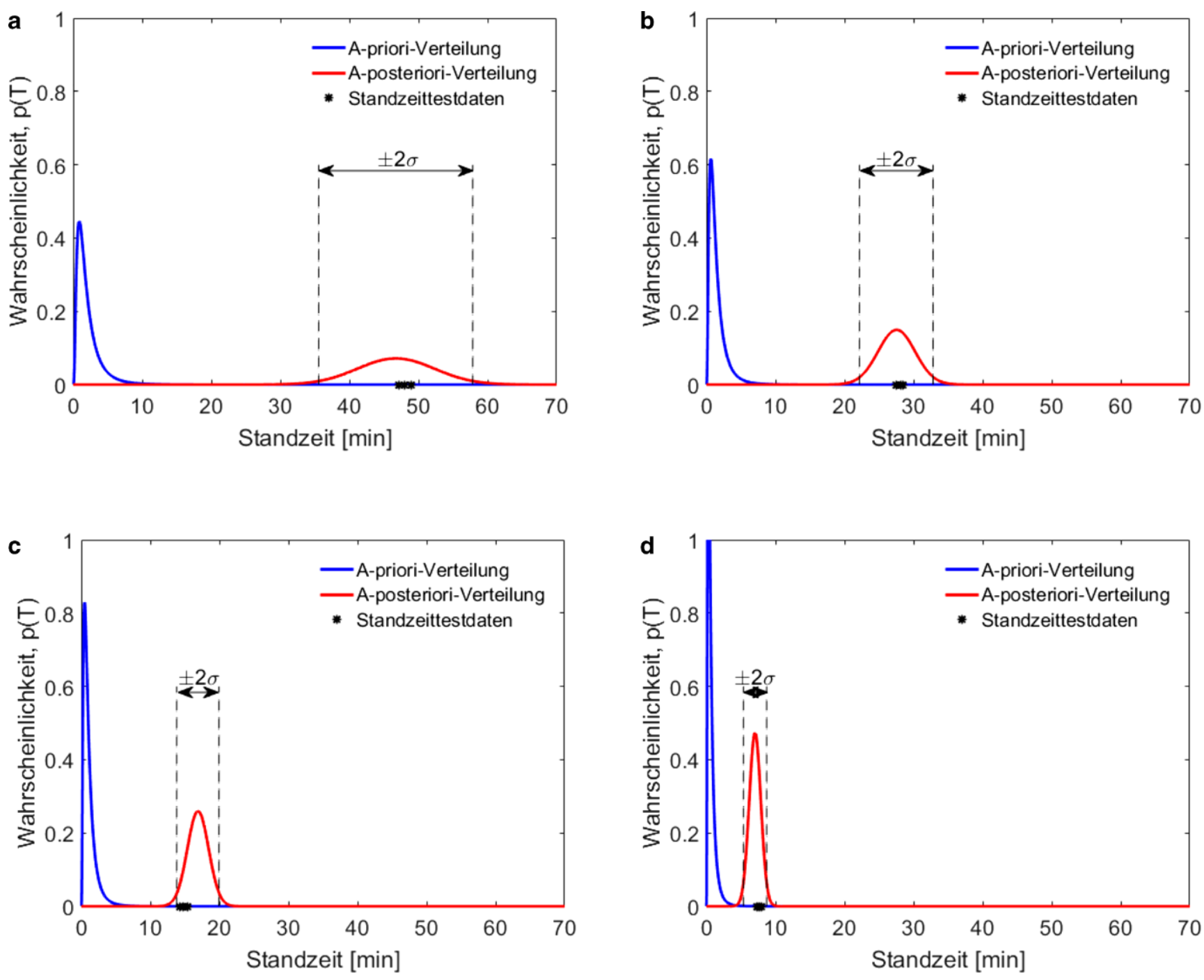

Abb. 10 A-posteriori-Funktionen mit Unsicherheitsintervallen der Standardabweichung von $\pm 2 \sigma$ unter Verwendung der Schnittgeschwindigkeiten: a 300 , b 325 , c 350 und d $400 \mathrm{~m} / \mathrm{min}$

ausreichend genau vorhersagen. Dies ist auf den höheren, zuvor ermittelten Fehlerprozentsatz zurückzuführen, der unter Verwendung der Gl. (9) in Tab. 2 angegeben wird.

In Tab. 3 sind die vorhergesagten Zuverlässigkeitswerte zusammengefasst. Sie entsprechen den gemessenen Werkzeugstandzeitdatenpunkten. Die Zuverlässigkeiten des Fräswerkzeugs bei den Schnittgeschwindigkeiten von $V_{c}=\{300$; $325 \mathrm{~m} / \mathrm{min}$ liegen näher am Funktionsmittelwert 0,5 aufgrund der genaueren Vorhersage der Testdatenpunkte bei diesen Schnittgeschwindigkeiten. Grundsätzlich nimmt die Zuverlässigkeit des Werkzeugs mit zunehmender Schnittlänge $a b$. Sie verringert sich mit Erhöhung der Schnittgeschwindigkeit. Abschließend ist festzustellen, dass die Ausfallwahrscheinlichkeit eines Schneidwerkzeugs zu einer bestimmten Schneidzeit mit Hilfe der Zuverlässigkeitsfunktion ermittelt werden kann.

\section{Zusammenfassung}

Die dargestellten Untersuchungen beinhalten die probabilistische Vorhersage der Werkzeugstandzeit und die Zuverlässigkeitsanalyse eines Fräswerkzeugs bei Schnittgeschwindigkeiten von 300 bis $400 \mathrm{~m} / \mathrm{min}$. Die Bayesianische MCMC-Methode wurde auf das Taylor-Modell angewendet, um die Wahrscheinlichkeitsverteilungen der Werkzeugstandzeit zu ermitteln. Die numerische Quantifizierung und Minimierung der Standzeitunsicherheit wurden unter Verwendung des Metropolis-Algorithmus der MCMC-Simulation durchgeführt. Die A-priori-Verteilungen wurden als Lognormalverteilungen modelliert. Die A-posteriori-Verteilungen wurden nach Aktualisierung der Modellparameter unter Verwendung von zwei gemessenen Werkzeugstandzeiten $T_{m}=\{7,6 ; 48\}$ min mit den jeweiligen Schnittgeschwindigkeiten von $V_{c}=\{300 ; 400\} \mathrm{m} / \mathrm{min}$ 

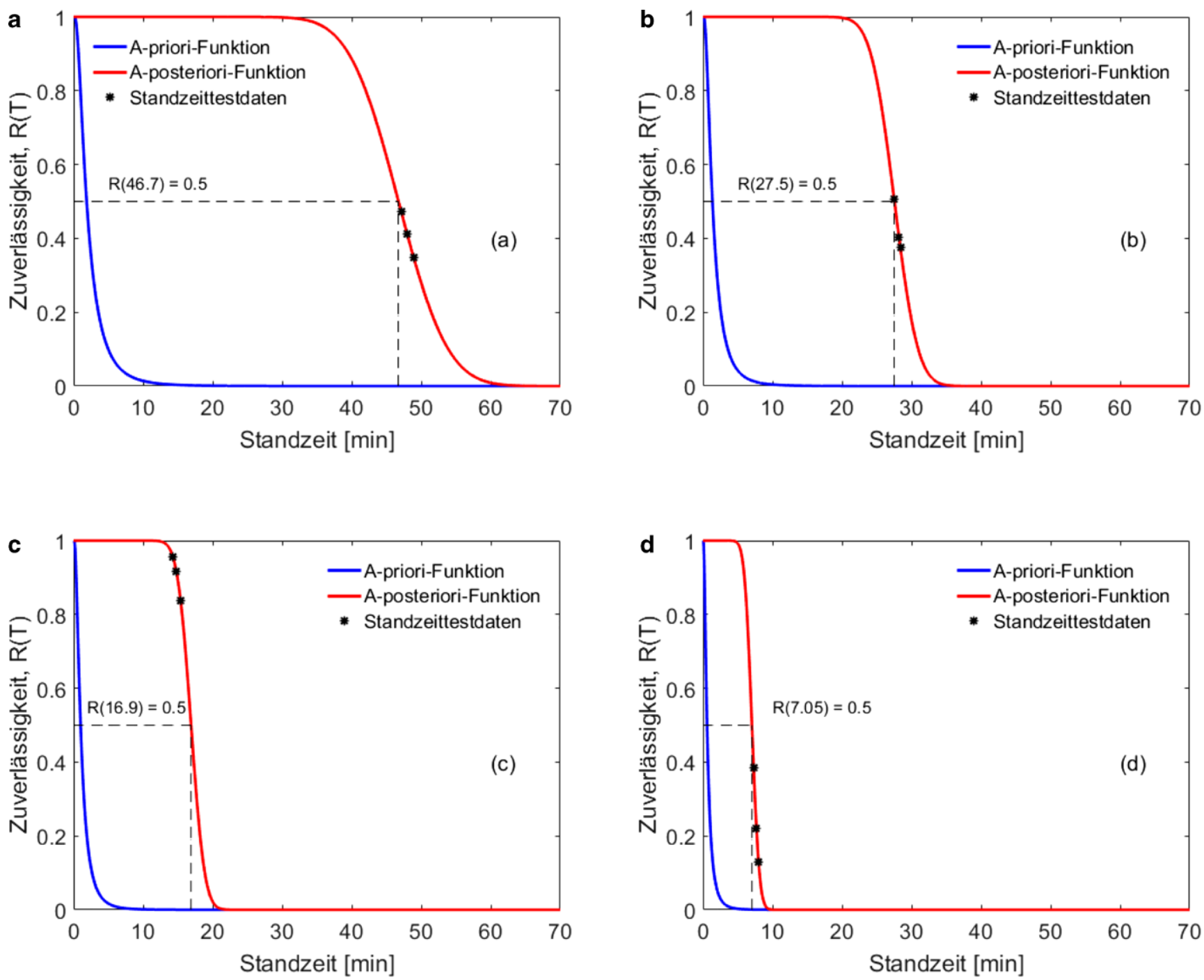

Abb. 11 A-posteriori-Zuverlässigkeitsfunktionen unter Verwendung der Schnittgeschwindigkeiten: a 300, b 325, c 350 und d 400 m/min

ermittelt. Anhand der Zuverlässigkeitsfunktion wurde abschließend eine Zuverlässigkeitsvorhersage zum Erreichen der festgelegten Verschleißmarkenbreite modelliert und vorhergesagt.

In dieser Forschung wurden die Unsicherheiten der Taylor-Modellparameter $C$ und $n$, sowie die Standzeitverteilungen bei allen Schnittgeschwindigkeiten minimiert. Die ermittelten A-posteriori-Verteilungen der Werkzeugstandzeit konnten alle Testdatenpunkte innerhalb der Unsicherheitsintervalle mit einem maximalen Vorhersagefehler von $18 \%$ (bei $V_{c}=350 \mathrm{~m} / \mathrm{min}$ ) vorhersagen. Des Weiteren konnten die Zuverlässigkeitsfunktionen die Testdatenpunkte um die Funktionsmittelwerte (mit Ausnahme der Schnittgeschwindigkeit von $V_{\mathrm{c}}=350 \mathrm{~m} / \mathrm{min}$ ) vorhersagen.

Die durchgeführte probabilistische Modellierung und Vorhersage der Standzeit und Zuverlässigkeit eines Fräswerkzeugs mittels der Bayesianischen Statistik ermöglicht die Vorhersage der Werkzeugstandzeit und die Bestimmung der Zuverlässigkeit eines Fräswerkzeugs mit einer gewissen Genauigkeit innerhalb eines definierten Unsicherheitsintervalls. Das entwickelte Modell ist dabei nicht allgemein gültig und nur für diesen Anwendungsfall bestimmt. Je nach Werkzeuggeometrien, Werkstückmaterialien und Schnittgeschwindigkeiten ist das Modell anzupassen. Beispielsweise müssen für jede Kombination aus Schneidwerkzeug und Werkstückmaterial sowie für eine neue Schnittgeschwindigkeit die Parameter $C$ und $n$ neu festgelegt und die Standzeit und die Zuverlässigkeitsfunktion neu berechnet werden.

Danksagung Der korrespondierende Autor dankt dem Karlsruhe House of Young Scientist (KHYS) des KIT zur Förderung des Forschungsaufenthalts an der University of North Carolina in Charlotte im Rahmen eines Stipendiums.

Funding Open Access funding provided by Projekt DEAL. 
Open Access Dieser Artikel wird unter der Creative Commons Namensnennung 4.0 International Lizenz veröffentlicht, welche die Nutzung, Vervielfältigung, Bearbeitung, Verbreitung und Wiedergabe in jeglichem Medium und Format erlaubt, sofern Sie den/die ursprünglichen Autor(en) und die Quelle ordnungsgemäß nennen, einen Link zur Creative Commons Lizenz beifügen und angeben, ob Änderungen vorgenommen wurden.

Die in diesem Artikel enthaltenen Bilder und sonstiges Drittmaterial unterliegen ebenfalls der genannten Creative Commons Lizenz, sofern sich aus der Abbildungslegende nichts anderes ergibt. Sofern das betreffende Material nicht unter der genannten Creative Commons Lizenz steht und die betreffende Handlung nicht nach gesetzlichen Vorschriften erlaubt ist, ist für die oben aufgeführten Weiterverwendungen des Materials die Einwilligung des jeweiligen Rechteinhabers einzuholen.

Weitere Details zur Lizenz entnehmen Sie bitte der Lizenzinformation auf http://creativecommons.org/licenses/by/4.0/deed.de.

\section{Literatur}

1. Arrazola PJ, Özel T, Umbrello D, Davies M, Jawahir IS (2013) Recent advances in modelling of metal machining processes. CIRP Ann 62(2):695-718. https://doi.org/10.1016/J.CIRP.2013.05.006

2. Attanasio A, Ceretti E, Rizzuti S, Umbrello D, Micari F (2008) 3D finite element analysis of tool wear in machining. CIRP Ann 57(1):61-64. https://doi.org/10.1016/J.CIRP.2008.03.123

3. Schulze V, Zanger F (2011) Development of a Simulation Model to Investigate Tool Wear in Ti-6Al-4V Alloy Machining. Adv Mater Res 223:535-544. https://doi.org/10.4028/www.scientific.net/ AMR.223.535

4. Yen Y-C, Söhner J, Lilly B, Altan T (2004) Estimation of tool wear in orthogonal cutting using the finite element analysis. J Mater Process Technol 146:82-91. https://doi.org/10.1016/S09240136(03)00847-1.

5. Denkena B, Tönshoff HK (2011) Spanen: Grundlagen. Springer, Berlin, Heidelberg

6. Karandikar JM, Abbas AE, Schmitz TL (2014) Tool life prediction using Bayesian updating. Part 1: milling tool life model using a discrete grid method. Precis Eng 38:9-17. https://doi.org/10.1016/J. PRECISIONENG.2013.06.006

7. Karandikar JM, Abbas AE, Schmitz TL (2014) Tool life prediction using Bayesian updating. Part 2: Turning tool life using a Markov Chain Monte Carlo approach. Precis Eng 38:18-27. https://doi.org/ 10.1016/J.PRECISIONENG.2013.06.007

8. Karandikar JM, Abbas AE, Schmitz TL (2013) Tool Life Prediction using Random Walk Bayesian Updating. Mach Sci Technol 17:410-442. https://doi.org/10.1080/10910344.2013.806103

9. Niaki FA, Ulutan D, Mears L (2015) Parameter Estimation Using Markov Chain Monte Carlo Method in Mechanistic Modeling of Tool Wear During Milling, in: Vol. 2 Mater. Biomanufacturing; Prop. Appl. Syst. Sustain. Manuf., ASME, p. V002T04A006. https://doi.org/10.1115/MSEC2015-9357.
10. Akhavan Niaki F, Ulutan D, Mears L (2016) Parameter Inference Under Uncertainty in End-Milling $\gamma^{\prime}$-Strengthened Difficult-to-Machine Alloy. J Manuf Sci Eng 138:61014. https://doi.org/10.1115/ 1.4033041

11. Bertsche B, Lechner G (1999) Einleitung, in: Zuverlässigkeit Im Maschinenbau. Springer Berlin Heidelberg, Berlin, Heidelberg, S 1-6 https://doi.org/10.1007/978-3-662-11004-1_1

12. Salonitis K, Kolios A (2014) Reliability assessment of cutting tool life based on surrogate approximation methods. Int J Adv Manuf Technol 71:1197-1208. https://doi.org/10.1007/s00170-013-55602

13. Salonitis K, Kolios A (2013) Reliability Assessment of Cutting Tools Life based on Advanced Approximation Methods. Procedia Cirp 8:397-402. https://doi.org/10.1016/J.PROCIR.2013.06.123

14. Lin WS (2008) The reliability analysis of cutting tools in the HSM. Processes 30:97-100

15. El Wardany TI, Elbestawi MA (1997) Prediction of tool failure rate in turning hardened steels. Int J Adv Manuf Technol 13:1-16. https://doi.org/10.1007/BF01179225

16. Salehi M, Schmitz TL, Copenhaver R, Haas R, Ovtcharova J (2019) Probabilistic sequential prediction of cutting force using kienzle model in orthogonal turning process. J Manuf Sci Eng Trans ASME https://doi.org/10.1115/1.4041710.

17. Salehi M, Schmitz TL, Copenhaver R, Haas R, Ovtcharova J (2018) Probabilistic Prediction of Cutting and Ploughing Forces using Extended Kienzle Force Model in Orthogonal Turning Process. Procedia Cirp 77:90-93. https://doi.org/10.1016/J.PROCIR.2018.08.228

18. Andrieu C, De Freitas N, Doucet A, Jordan MI (2003) An introduction to MCMC for machine learning. Mach Learn 50:5-43. https:// doi.org/10.1023/A:1020281327116

19. Karandikar JM (2013) The fundamental application of decision analysis to manufacturing, University of North Carolina at Charlotte. http://libres.uncg.edu/ir/uncc/f/Karandikar_uncc_0694D_ 10487.pdf

20. Salehi M, Wald G, Jeretin-Kopf M, Blum M, Schmitz TL, Haas R, Ovtcharova J (2019) Entwicklung eines probabilistischen Modells zur Vorhersage von Schnittkräften beim unterbrochenen Drehen von Inconel 718. Forsch Im Ingenieurwes. https://doi.org/10.1007/ s10010-019-00298-w

21. Salehi M (2019) Bayesian-Based Predictive Analytics for Manufacturing Performance Metrics in the Era of Industry 4.0. https://doi. org/10.5445/ksp/1000091806. Zugegriffen: 10.10.2019

22. Roberts GO, Rosenthal JS (2001) Optimal scaling for various Metropolis-Hastings algorithms. Stat Sci 16:351-367. https://doi.org/ $10.1214 / \mathrm{ss} / 1015346320$

23. Hoff PD (2009) A first course in Bayesian statistical methods. Springer, New York https://doi.org/10.1007/978-0-387-92407-6

24. Niaki FA (2016) A Probabilistic-Based Approach to Monitoring Tool Wear State and Assessing Its Effect on Workpiece Quality in Nickel-Based Alloys, Clemson University. http://tigerprints. clemson.edu/all_dissertations/1858

25. Geweke J (1992) Evaluating the accuracy of sampling-based approaches to the calculation of posterior moments. Bayesian Stat. 4:169-193. 